\title{
Functional contributions of glutamate transporters at the parallel fibre to Purkinje neuron synapse-relevance for the progression of cerebellar ataxia
}

\author{
Emmet M Power and Ruth M Empson*
}

\begin{abstract}
Background: Rapid uptake of glutamate by neuronal and glial glutamate transporters (EAATs, a family of excitatory amino acid transporters) is critical for shaping synaptic responses and for preventing excitotoxicity. Two of these transporters, EAAT4 in Purkinje neurons (PN) and EAAT1 in Bergmann glia are both enriched within the cerebellum and altered in a variety of human ataxias.

Results: PN excitatory synaptic responses and firing behaviour following high frequency parallel fibre (PF) activity commonly encountered during sensory stimulation in vivo were adversely influenced by acute inhibition of glutamate transporters. In the presence of a non-transportable blocker of glutamate transporters we observed very large amplitude and duration excitatory postsynaptic currents accompanied by excessive firing of the PNs. A combination of AMPA and mGluR1, but not NMDA, type glutamate receptor activation powered the hyper-excitable PN state. The enhanced PN excitability also recruited a presynaptic mGluR4 dependent mechanism that modified short term plasticity at the PF synapse.

Conclusions: Our findings indicate that reduced glutamate transporter activity, as occurs in the early stages of some forms of human cerebellar ataxias, excessively excites PNs and disrupts the timing of their output. Our findings raise the possibility that sustaining cerebellar glutamate uptake may provide a therapeutic approach to prevent this disruption and the glutamate excitotoxicity-induced PN death that signals the end point of the disease.
\end{abstract}

Keywords: Cerebellum, Glutamate transporters, Parallel fibres, Plasticity, Facilitation, Ataxia

\section{Background}

The timing of excitatory synaptic responses at mammalian glutamatergic synapses relies upon the rapid recruitment of postsynaptic glutamate receptors and the rate of clearance of glutamate from the synaptic cleft. Glutamate clearance relies upon glutamate transporter carrier proteins (excitatory amino acid transporters, EAATs) located in close proximity to the synapse [1] that couple glutamate movement with the $\mathrm{Na}^{+}$electrochemical gradient, $\mathrm{H}^{+}$movement and a countertransported $\mathrm{K}^{+}$ion [2]. Furthermore, these transporters also behave like ligand gated ion channels, since glutamate activates their

\footnotetext{
* Correspondence: ruth.empson@otago.ac.nz

Department of Physiology, Brain Health Research Centre, University of Otago School of Medical Sciences, PO Box 56, 9054 Dunedin, New Zealand
}

inbuilt chloride conductance [3]. The functional importance of the glutamate transporters in the cerebellum is highlighted by the appearance of mutations in cerebellarenriched EAAT1 in human episodic ataxia type 6 [4-6], the down-regulation of EAATs in animal models of the human spinocerebellar ataxias SCA1, 5 and 7 [7-10] and the mildly ataxic phenotype of mice lacking EAAT1 [11]. Interestingly, in episodic ataxia type 6, a mutation in EAAT1 enhances this transporter's anion conductance [4] suggesting a potential role for these anion channels in disease.

The main output neurons of the cerebellar cortex, the Purkinje neurons (PN), are highly enriched for EAAT4 [12] present exclusively within the PN somatodendritic compartment [12] and perisynaptic regions [13]. This 
location means it powerfully influences both climbing fibre (CF) and parallel fibre (PF) synapse responses [14]. Furthermore, since EAAT4 also exhibits the largest glutamate activated chloride (anion) conductance it also has the potential to directly modify the excitability of the PN dendrite [15]. EAAT4 expression is heterogeneous across the cerebellar cortex and enhanced levels coincide with the expression of zebrin II-positive stripes [13] and elevated glutamate release from CF terminals [16]. Under certain conditions the elevated EAAT4 in zebrin IIpositive zones may exert a neuroprotective influence against PN glutamate excitotoxicity [17]. More widely, EAAT4 influences extrasynaptic transmission from PFs to adjacent Bergmann glia [18] and controls metabotropic glutamate type 1 receptor-mediated (mGluR1) synaptic events at PF and CF synapses to influence plasticity [19-21]. Without EAAT4, glutamate released by CF activation can even escape far enough to recruit basket cells and drive intersynaptic GABAergic transmission [22]. EAAT3 (EAAC1) is less abundant in the cerebellum, but like EAAT4, it locates to the somatodendritic compartment of the PN [12]. Interestingly EAAT3 (also known as EAAC1) is negatively modulated by its interacting protein, glutamate transporter-associated protein 3-18 (GTRAP3-18) [23] and is known to transport cysteine into cells to produce neuroprotective glutathione [24,25].

The cerebellar cortex also richly expresses the astrocytic forms of the glutamate transporters, particularly EAAT1 (GLAST) and EAAT2 (GLT-1). Of the two, EAAT1 is most enriched in the Bergmann glial cells [26] and like EAAT4 also resides in close proximity to the PN somatodendritic compartment [1]. Uptake of glutamate by EAAT1 limits cross talk across cerebellar synapses; it controls the amplitude and time course of both CF-and PF-evoked AMPA receptor-mediated synaptic transmission [27] by preventing glutamate escape. EAAT1 mediated glutamate uptake also maintains the one to one innervation of the PN by the CF [28] and the independent operation of PF synapses [29]. Furthermore, during CF activation EAAT1 controls the spread of glutamate to nearby post-synaptic NMDA receptors on the PN spine [30] and also to presynaptic NMDA receptors on GABAergic terminals thereby also influencing local synaptic inhibition [31].

Here we show how the non-transportable blocker of EAATs 1-5, TBOA, (DL-threo-beta-benzyloxyaspartate, hereafter referred to as TBOA) adversely influences the behaviour of the PN and the PF-PN synapse, and discuss how these mechanisms might influence cerebellar synapse behaviour in the early stages of cerebellar ataxias. We used brief bursts of high frequency physiologically relevant PF activity to activate the PN (200 Hz EPSC), and show how TBOA enhanced the amplitude and duration of the PN EPSC in an mGluR1 dependent and independent manner. This enhanced depolarisation prolonged burst firing of the $\mathrm{PN}$ in response to PF input, even when mGluR1 mediated excitation was blocked. The excess glutamate made available by TBOA also recruited abnormal PF presynaptic mGluR4 signalling that modified the behaviour of the synapse. We predict that these mechanisms have the potential to contribute to mistimed cerebellar output during the onset and progression of some human cerebellar ataxias. Our findings also highlight the importance of developing ways to sustain cerebellar glutamate uptake as a useful early therapy to prevent the progression of cerebellar ataxia.

\section{Results}

Inhibition of glutamate transport enhanced high frequency stimulus-evoked parallel fibre EPSCs and revealed a slow EPSC with mGluR1 dependent and independent components

A burst of ten, high frequency $(200 \mathrm{~Hz})$ stimulations to the PFs aimed to mimic the in vivo behaviour of PFs [32] and evoked a large amplitude long-lasting EPSC in cerebellar PNs, Figure 1 and Figure 2A, hereafter called the high-frequency PF EPSC. To inhibit glutamate transport we used $50 \mu \mathrm{M}$ TBOA. This concentration of TBOA significantly and reversibly enhanced both the peak amplitude and the duration of the high-frequency PF EPSC, Figure 2B. These changes occurred in the absence of any changes in the series resistance, input resistance or holding current of the PN (see Methods below). TBOA application also revealed a larger amplitude slow component to the high-frequency PF EPSC that was reduced by the broad spectrum mGluR1 antagonist, MCPG (0.2 mM), leaving a smaller amplitude, slow EPSC remaining, Figure 2B. In the presence of the AMPA and Kainate receptor (KA-R) antagonist CNQX (6-cyano-7-nitroquinoxaline-2,3-dione) and TBOA and MCPG (see also Table 1 for explanation),the fast peak amplitude of the high-frequency PF EPSC reduced from $980 \pm 167 \mathrm{pA}$ in control to $112 \pm 36 \mathrm{pA}, \mathrm{n}=4, \mathrm{P}<0.001$, $t$-test, whilst the slower component EPSC reduced from $65 \pm 16 \mathrm{pA}$ to $2 \pm 1.2 \mathrm{pA}$ (see Figure $2 \mathrm{~A}$ ). This result indicated that the majority of the TBOA-enhanced highfrequency PF EPSC resulted from AMPA-R ( $\alpha$-Amino-3hydroxy-5-methyl-4-iso xazolepropionic acid receptors) and KA-R activation. The NMDA (glutamate) receptor antagonist APV $(n=6)$ did not influence the amplitude or duration of the high-frequency PF EPSC in the presence of TBOA (mean values of the EPSC changed from $771 \pm 66 \mathrm{pA}$ for control to $1087 \pm 88.3 \mathrm{pA}$ in TBOA, $\mathrm{P}<$ 0.05 , one way ANOVA, but remained unchanged at $1128 \pm 124 \mathrm{pA}$ in the presence of TBOA and APV, not significant in one way ANOVA multiple comparison). We also observed reversibility of the effects of $50 \mu \mathrm{M}$ TBOA in 4 cells 15 minutes after wash back; mean 

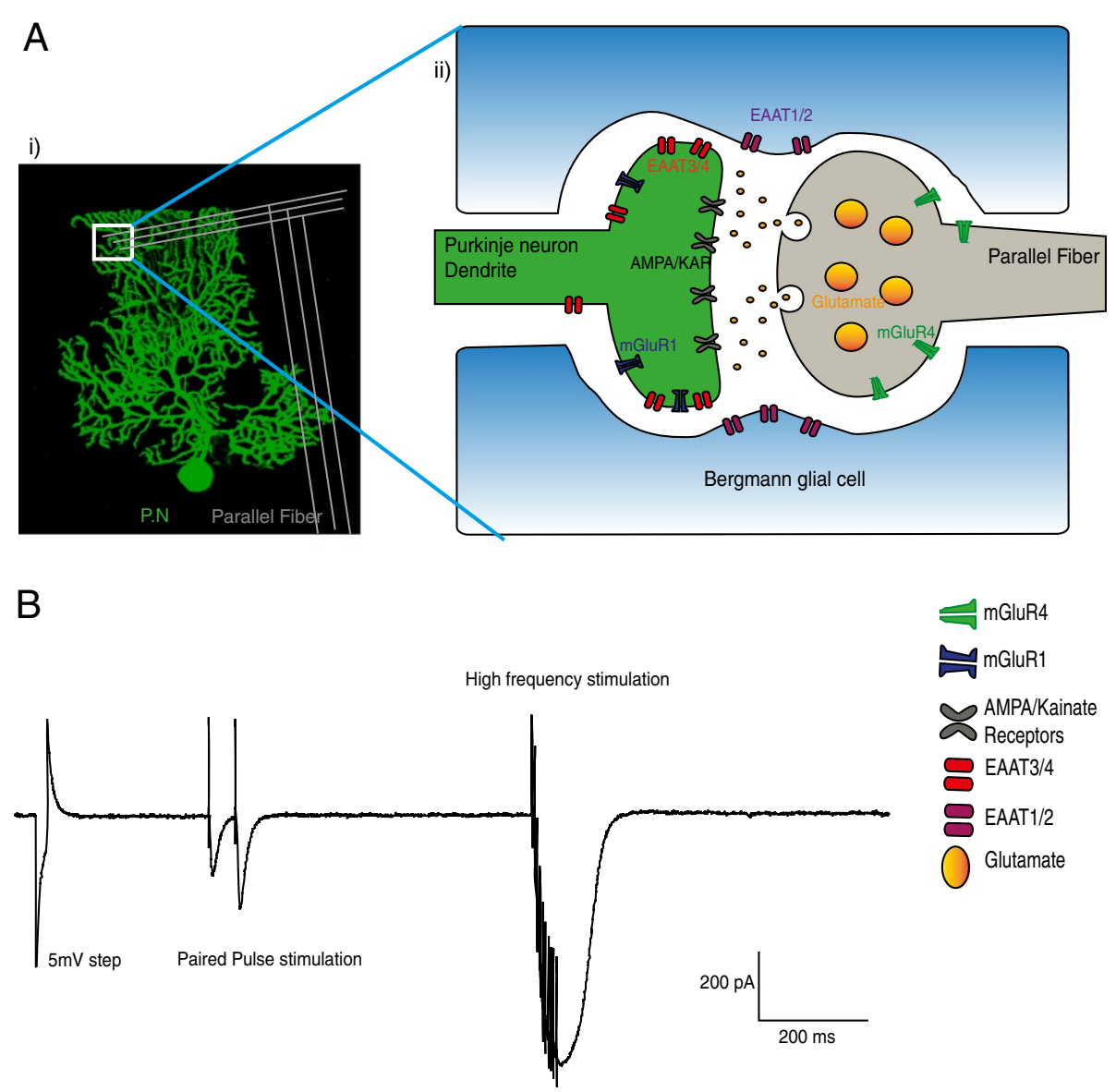

Figure 1 Location of all the major transporters and receptors at a PF-PN synapse. (A)(i) A biocytin filled Purkinje neuron post-hoc stained with streptavidin Alexa 488. (ii) A representative image of a Parallel fibre Purkinje neurons synapse showing the approximate locations of the major transporters and receptors targeted in this study. Parallel fibre synapses are located on the outer dendrites of Purkinje neurons, highlighted by the white box in (Ai). (B) An example trace showing our stimulation and recording protocol. We used a $5 \mathrm{mV}$ step to calculate series and input resistance, followed by application of a pair of closely spaced stimuli to the parallel fibres and a high frequency burst stimulation (10 stimuli at $200 \mathrm{~Hz}$ ), a protocol we repeated every 30 seconds.

values of the amplitude and duration of the $200 \mathrm{~Hz}$ EPSC changed from $487 \pm 64 \mathrm{pA}$ to $674 \pm 67 \mathrm{pA}$ in TBOA and back to $503 \pm 65 \mathrm{pA}\left(\mathrm{F}_{2,11}=14.7, \mathrm{P}<0.05\right.$, one way ANOVA) and $291 \pm 47 \mathrm{~ms}$ to $615 \pm 118 \mathrm{~ms}$ and back to $379 \pm 55 \mathrm{~ms}$ respectively $\left(\mathrm{F}_{2,11}=4.4, \mathrm{P}<0.05\right.$, one way ANOVA). The time constant of the recovery of the $2^{\text {nd }}$ EPSC was also reversible, mean values changed from $19.3 \pm 1.7 \mathrm{~ms}$ to $30 \pm 3.2$ and back to $22.9 \pm 2.5 \mathrm{~ms}$ $\left(\mathrm{F}_{2,11}=4.6, \mathrm{P}<0.05\right.$, one way ANOVA $)$.

Subtraction of the high frequency PF EPSC signal from the same signal in the presence of TBOA allows calculation of a difference current, seen in Figure 2A. This provides a quantitative index of the influence of glutamate transporter activity on the EPSC, so that without a functioning transporter we observe a greater current consistent with greater availability of glutamate at the synapse. The difference current also readily distinguishes the two distinct phases of the TBOA-enhanced high-frequency
PF EPSC as additional AMPA-R, KA-R and mGluR1 receptors are recruited as a consequence of the greater availability of glutamate. The initial phase, that influences the peak of the EPSC, peaks at around $150 \mathrm{~ms}$ post stimulation, while the slower phase peaks approximately $400 \mathrm{~ms}$ later. MCPG reduced the slow phase leaving a small but measurable slow current, Figure $2 \mathrm{C}$, but did not significantly influence the early phase suggesting that this is mediated exclusively by AMPA and KA-Rs, and as shown in Figure 2A, further addition of AMPA-KA receptor antagonist, CNQX, blocked all current.

Our findings confirm that a high frequency burst of PF activity is sufficient to activate glutamate uptake by TBOA sensitive glutamate transporters, consistent with a large body of previous literature. The difference current implies that under our experimental conditions, uptake peaks approximately $100 \mathrm{~ms}$ after stimulation and continues for several hundred milliseconds thereafter. The 


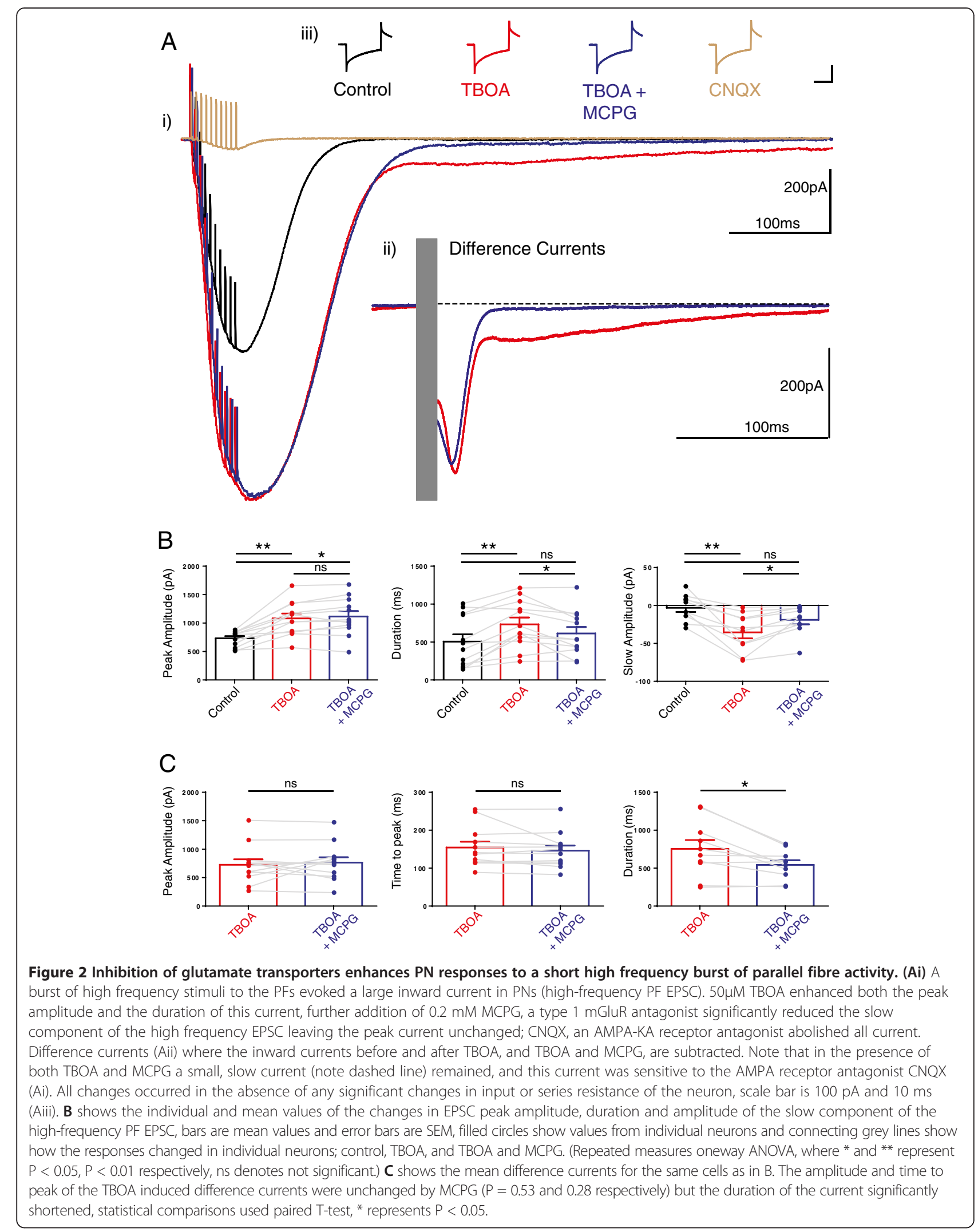


Table 1 Table of major transporters and receptors involved in this study

\begin{tabular}{lll}
\hline Receptor/Transporter & Location & Antagonist \\
\hline EAAT1 & Bergmann Glia & TBOA \\
EAAT2 & Bergmann Glia & TBOA \\
EAAT3 & Purkinje neuron (PN) & TBOA \\
EAAT4 & Perisynaptic on PN & TBOA \\
AMPA/Kainate & PN, postsynaptic density (PSD) & CNQX \\
mGluR1 & PN, edge of PSD & (S)-MCPG \\
mGluR4 & Parallel fiber presynapse & MPPG
\end{tabular}

$\mathrm{A}$ list of major transporters and receptors used in this study along with their associated antagonists and locations. DL-TBOA $=$ DL-threo- $\beta$-Benzyloxyaspartic acid, CNQX = 6-cyano-7-nitroquinoxaline-2,3-dione,

(S)-MCPG = (S)-a-Methyl-4-carboxyphenylglycine,

MPPG = (RS)-a-Methyl-4-phosphonophenylglycine.

consequence of inhibition of glutamate uptake is that PF activation encourages a significant and long lasting depolarisation of the PN via the recruitment of AMPA, KA and mGluR1 receptors.

\section{Inhibition of glutamate transport enhanced Purkinje neuron firing in response to high frequency PF input that was refractory to the mGluR1 antagonist MCPG}

In order to determine the consequence of reduced glutamate transport for PN excitability we next assessed how TBOA influenced spontaneous and burst evoked action potential firing behaviour. Since the difference currents (Figure 2A) indicate that the largest influence of reduced transporter activity occurs approximately $100 \mathrm{~ms}$ after the last burst and that the slower component peaks around $400 \mathrm{~ms}$ later, we analysed action potential firing over these times and compared them with pre stimulation firing.

As shown in Figure 3 TBOA significantly increased the firing frequency at both $0-100 \mathrm{~ms}$ and $100-500 \mathrm{~ms}$ after the stimulation, compared with basal, pre stimulation firing frequency (Row factor in two way ANOVA $\left.F_{2,24}=96.8, P<0.0001\right)$. Surprisingly, removal of mGluR1 mediated receptor activation (by MCPG) did not influence the post stimulation firing frequency (not significant in two way ANOVA multiple comparisons). TBOA also did not significantly influence the firing properties of the PN measured in the 500 ms before the PF burst, consistent with the lack of any significant change in holding current of individual PNs by $50 \mu \mathrm{M}$ TBOA (see Methods).

Inhibition of glutamate transport reduced paired pulse facilitation at the PF synapse via an mGluR4-mediated mechanism

Although reduced glutamate uptake clearly amplified PN excitability per se (Figure 2A) the excess glutamate under these conditions could also influence PF-PN synapse behaviour through presynaptic mechanisms. To test this we used the fact that the low release probability PF synapse exhibits the phenomenon of paired pulse facilitation (PPF). In this phenomenon, elevated residual presynaptic calcium encourages greater glutamate release in response to the second of a pair of closely spaced stimulations. Changes in presynaptic properties that reduce calcium influx are reliably predicted to reduce glutamate release and to enhance the facilitation of the second EPSC.

As seen in Figure 4, TBOA increased PPF at the PF synapse. This result implies that excess glutamate availability reduced the probability of glutamate release at this synapse. Importantly, in 7/11 cells we observed this TBOA-enhanced PPF in the absence of any change in the amplitude of the first EPSC, as seen in Figure 4A. In the remaining $4 / 11$ cells TBOA enhanced the $1^{\text {st }}$ EPSC and PPF, shown in Figure 4A, but we excluded these cells since interpretation becomes more complex under these circumstances. Note also that TBOA prolonged the recovery of the second of the pair of EPSCs, see Figure $4 \mathrm{~A}$, consistent with a slowed recovery of postsynaptic glutamate clearance.

In order to search for the mechanism underlying the TBOA-enhancement of PPF we omitted the high frequency stimulation that we routinely applied within our protocol (shown in Figure 1). Previous studies indicate that cannabinoid plasticity at this synapse only occurs in the presence of the high frequency bursts that mimic in vivo PF behaviour [33]. Under these conditions a brief burst of PF activity can cause mGluR1 activation and a localised increase in $\mathrm{Ca}^{2+}$ concentration. This subsequently leads to an endocannabinoid mediated suppression of glutamate release from the PF via the CB1 receptor $[34,35]$. Similarly, when we omitted the high frequency stimulation TBOA failed to influence PPF ( $\mathrm{P}=0.16, \mathrm{n}=4$, paired $t$-test). This strongly supported the idea that an activity-dependent plasticity mechanism contributed to the TBOA-induced enhancement of the PPF. However, we ruled out an mGluR1-mediated postsynaptic generation of a retrograde signal (such as from cannabinoid release from the $\mathrm{PN},[33]$ ) since TBOA continued to enhance PPF in the presence of $0.2 \mathrm{mM}$ MCPG, the type 1 metabotropic glutamate receptor antagonist. Mean PPF was unchanged from $1.72 \pm 0.07$ to $1.73 \pm 0.06, \mathrm{n}=12$, not significant in multiple comparisons in a one-way ANOVA. Similarly, we excluded an action from presynaptic NMDA receptors at PFs [36] since the enhanced PPF persisted in the presence of TBOA + APV (an NMDA receptor antagonist). Mean values remained unchanged from $1.63 \pm 0.04$ to $1.64 \pm$ $0.05, \mathrm{n}=6$, not significant in multiple comparisons in a one-way ANOVA. 


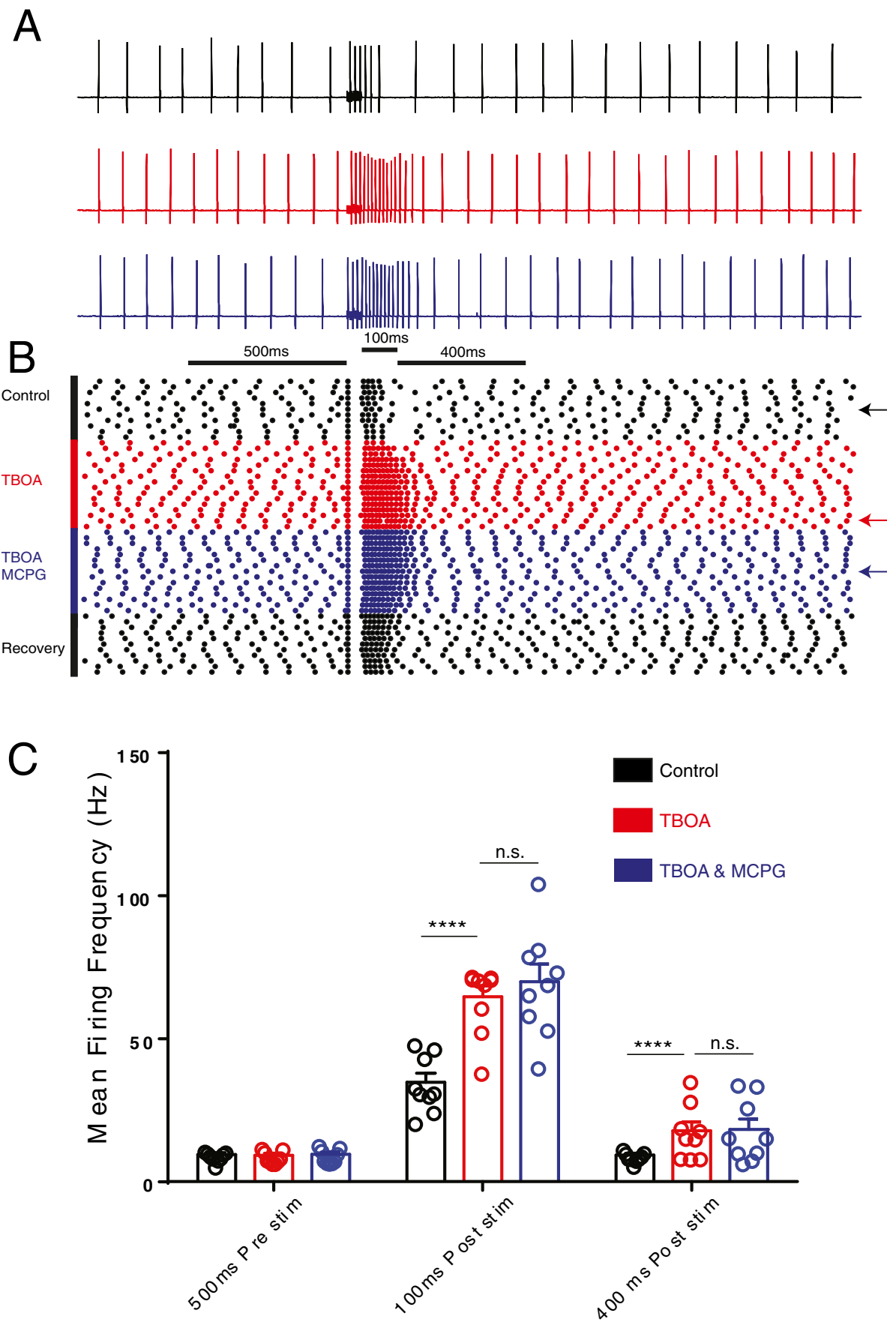

Figure 3 Inhibition of glutamate transporters enhances immediate and prolonged burst firing of PNs in response to a short high frequency burst of parallel fibre activity. A, example cell-attached recording from a PN reveals how action potential firing changes during the application of $50 \mu \mathrm{M}$ TBOA and $50 \mu \mathrm{M}$ TBOA in the presence of the mGluR antagonist $0.2 \mathrm{mM} \mathrm{MCPG}$. Horizontal bar represents $200 \mathrm{~Hz}$ high frequency stimulation to PFs. B, shows a raster plot for the cell shown in A, each sweep (horizontal dots represent each action potential in the sweep) occurred at an interval of 30 seconds during the application of TBOA and TBOA and MCPG (left, vertical bars). Note the enhanced firing after the burst in the presence of TBOA and the inability of MCPG to reduce this. Arrows represent the raster plot for the traces shown above. C, bars show mean values (error bars are sem) of action potential firing frequency obtained from a 500 ms epoch just before stimulation, compared with action potential firing frequency at the peak of the TBOA-induced $200 \mathrm{~Hz}$ EPSC (during the $100 \mathrm{~ms}$ after the end of the stimulation) and later during the $400 \mathrm{~ms}$ after the stimulation when the slower, mGluR1 and EAAT4 dependent phase of the $200 \mathrm{~Hz}$ EPSC is active. **** represents $P<0.0001$, two way ANOVA, ns is not significant. 

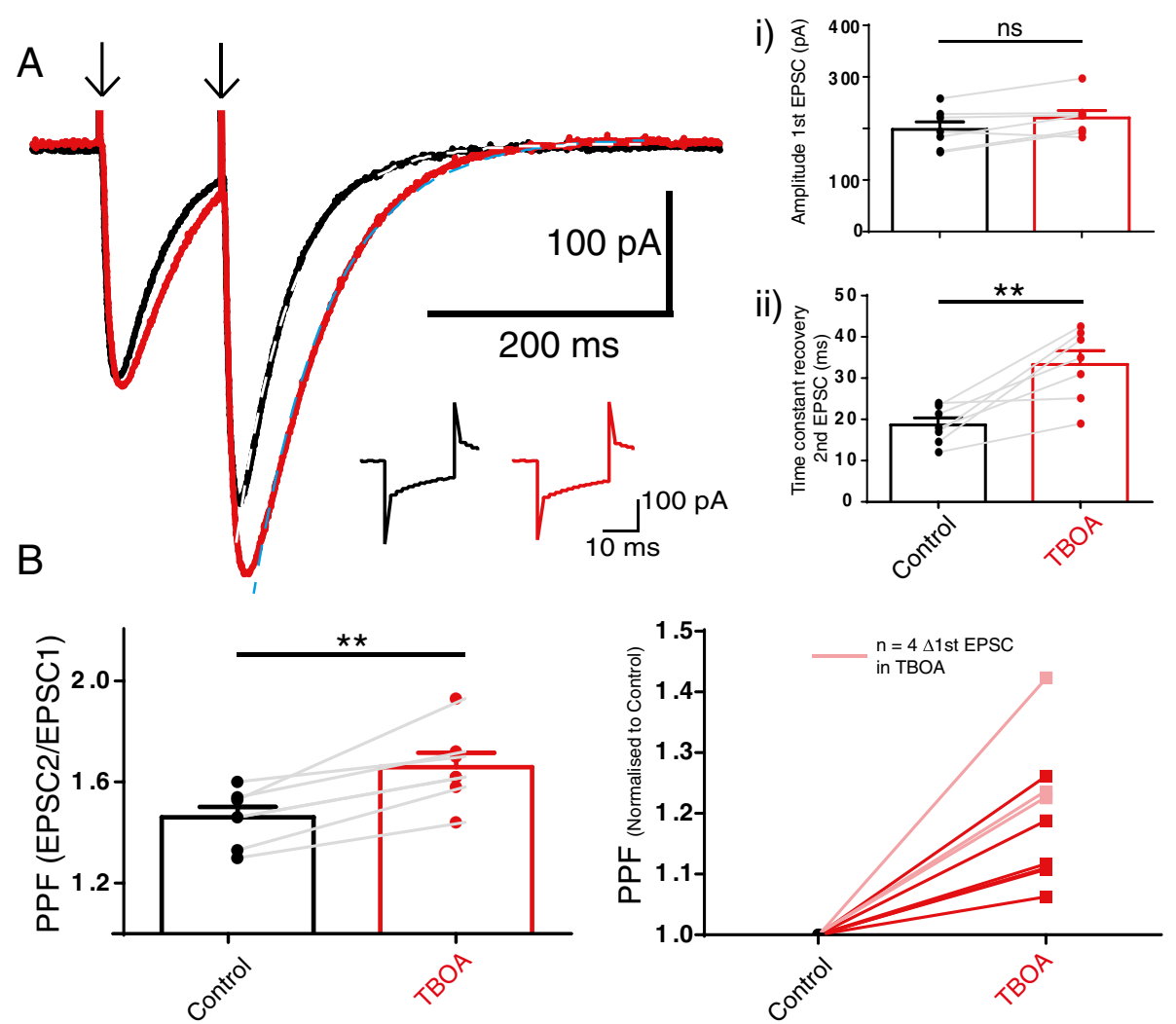

Figure 4 Inhibition of glutamate transporters enhances paired pulse facilitation at the PF-PN synapse and prolongs the decay of the excitatory post synaptic current, EPSC. A, shows an example recording from a PN showing EPSCs evoked with a pair of closely timed single stimulations to the PFs (downward arrows). Note the enhancement of the amplitude of the second EPSC upon application of $50 \mu \mathrm{M}$ TBOA and the concomitant increase in duration of the second EPSC, in the absence of any change in the amplitude of the first EPSC (in 7/11 cells shown in $A$ (i) where bars are mean \pm SEM, ns is not significant in Student's paired $t$-test). Dashed lines in A represent single exponential fits to estimate the time constant of recovery of the second EPSC; mean values (error bars are SEM) shown in A(ii) ** represents P $<0.01$ Student's paired t-test. Inset traces indicate no change in series resistance or input resistance by TBOA, see text, scale bar is $100 \mathrm{pA}$ and $10 \mathrm{~ms}$. B shows the change in mean absolute values of PPF in Control and after $50 \mu \mathrm{M}$ TBOA, bars are mean \pm SEM, *** represents $P<0.01$ Student's paired $t$-test. Left panel shows PPF change normalised to control for the 7/11 cells, bold colour, with remaining cells where the first EPSC increased, and PPF also increased, after TBOA, shown in a transparent shade.

We therefore turned to mGluR4, a metabotropic glutamate receptor known to be expressed presynaptically at PFs [37] and known to depress their glutamate release probability [38]. As seen in Figure 5A, $25 \mu \mathrm{M}$ MPPG, an antagonist of mGluR4 at PFs [39] abolished the TBOAenhanced PPF. MPPG did not influence the TBOAinduced slower recovery of the second of the pair of EPSCs nor did it prevent the enhanced amplitude of the $200 \mathrm{~Hz}$ evoked large EPSC (both $\mathrm{P}>0.05$ in multiple comparisons from one way ANOVAs), see also Figure $5 \mathrm{~B}$. This strongly supports the action of excess glutamate at PF presynaptic mGluR4 receptors. MPPG also did not itself influence PPF, see Figure 5A, or the amplitude of the first EPSC (mean values were unchanged from control $204 \pm 26 \mathrm{pA}$ to $217 \pm 32$ in MPPG and $239 \pm 39 \mathrm{pA}$ in TBOA and MPPG, not significant in a one way ANOVA). Although KA-R also contribute to enhanced facilitation following high frequency PF stimulation [40] we did not observe any remaining PPF enhancement in the presence of TBOA and MPPG suggesting that presynaptic KA-R were unlikely to be recruited under our conditions.

\section{Discussion}

Our findings illustrate that even a short period of reduced glutamate uptake adversely amplified synaptic responses at the PF-PN synapse to an extent that was sufficient to prolong PN firing output. Furthermore, reduced glutamate uptake also changed the timing and behaviour of the PF-PN synapse by the action of excess glutamate at presynaptic mGluR4 receptors.

The amplification of the PF-EPSC by TBOA is consistent with previous literature showing the importance of glutamate uptake at this and the climbing fibre (CF)-PN synapse [14-18]. The difference currents in Figure 2 show that glutamate uptake amplified both the fast and 

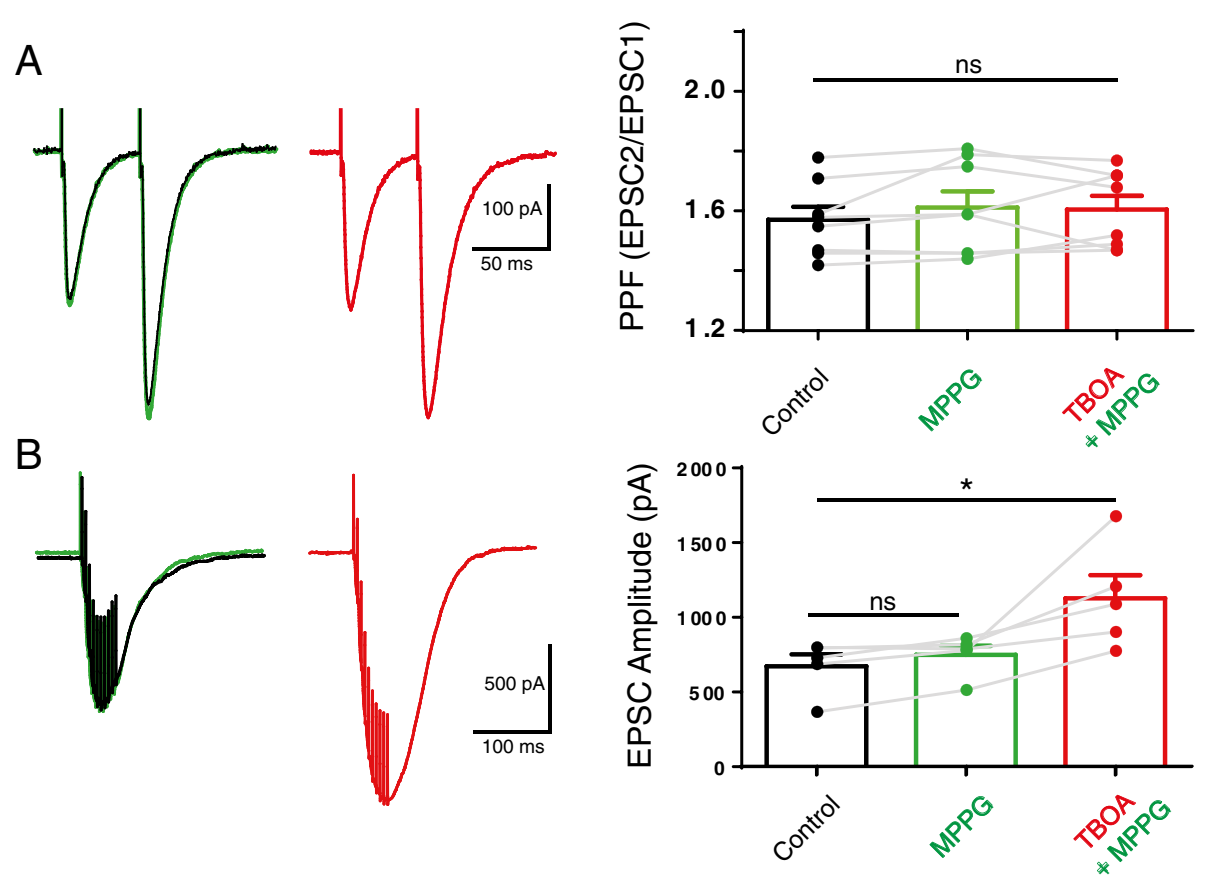

Figure 5 The mGluR4 (type III) receptor antagonist MPPG specifically prevented the change in paired pulse facilitation (PPF) upon inhibition of glutamate uptake. A shows that application of $50 \mu \mathrm{M}$ TBOA in the presence of the mGluR4 antagonist MPPG no longer changed the PPF and that PPF was also not significantly changed by $25 \mu \mathrm{M} \mathrm{MPPG}$ alone. The graph shows the individual and mean values of the changes in PPF, bars are mean values and error bars are SEM, filled circles show values from individual neurons and connecting grey lines show response changes in individual neurons. The statistical comparison used a repeated measures oneway ANOVA where ns denotes not significant, $F_{2,23}=1.1$, $P=0.35$. Importantly the first EPSC was also unchanged (data not shown) in these experiments, $F_{2,23}=4.4, P=0.08$. TBOA still increased the time course of the recovery of the second of the pair of EPSCs, consistent with its postsynaptic action independent of the presence of MPPG, mean values changed from $14.7 \pm 1.6 \mathrm{~ms}$ in control cells, to $14.2 \pm 1.5 \mathrm{~ms}$ in MPPG alone treated cells to $21.7 \pm 3.4 \mathrm{~ms}$ in MPPG and TBOA treated cells $\left(F_{2,23}=11.8, \mathrm{P}<0.05\right.$ repeated measures one way ANOVA). B shows for the same example cell as in A that the amplitude of the $200 \mathrm{~Hz}$ EPSC also increased in the presence of TBOA and MPPG, the graph is as for A above with a repeated measures oneway ANOVA where $F_{2,14}=8.7, P<0.05$ and multiple comparisons where * represents $\mathrm{P}<0.05$ and ns denotes not significant.

slow components of the PF response. The fast component likely represents excess glutamate acting at AMPA/ $\mathrm{KA}$ receptors since it was abolished by CNQX. Likewise, the slow component was abolished by the type 1 metabotropic glutamate receptor antagonist MCPG, as shown by others at both PF and CF synapses $[14,19,20]$, however a significant slow inward current remained. The fact that the AMPA-R antagonist CNQX abolished this slow MCPG-insensitive inward current in the PN indicates that it is a consequence of glutamate acting at glutamate receptors (including low affinity KA-R) [27]. Since previous work indicates that it is also absent in EAAT4 knockout mice [41] we propose that the slow current is driven by the opening of glutamate receptors (probably K-AR) as a consequence of excess available glutamate following EAAT4 inhibition by TBOA. The small, slow nature of the current is consistent with EAAT4 actions since EAAT4 is a high affinity glutamate transporter normally responsible for clearing the last remaining glutamate after PF stimulation; hence this slow current is most apparent in the presence of TBOA.
In accordance with this previous literature we also observed a significant overlap between the mGluR insensitive, CNQX sensitive slow current (EAAT4 action) and the mGluR1-sensitive, CNQX insensitive current (mGluR1 action), Figure 2A. The overlap of timing of these two components is consistent with the idea that mGluR1 responses are limited in PNs within zebrin IIpositive zones [20] where EAAT4 expression is highest [13] but are facilitated in PNs from EAAT4 knockout mice [18].

EAAT4 is known to have a large anion conductance compared to other EAATs [42], these anion currents, under resting conditions may inhibit cell excitability. It has also been speculated that EAAT4s hyperpolarising action could counteract the depolarising action of other EAATs, namely EAAT3. However, another report by Melzer [43] showed that during large depolarisations the EAAT4 transporter may become cation permeable and cause an excitatory inward current. A further consideration is that whilst EAAT4 currents may directly influence the local membrane potential in the PN dendrites 
they are unlikely to be directly responsible for the slow inward current recorded from the PN soma seen here, especially considering its susceptibility to CNQX.

\section{Inhibition of glutamate uptake prolongs Purkinje neuron firing output in response to PF input}

We expected the sizeable fast and slow excitatory inward currents evoked by high-frequency PF stimulation in the presence of TBOA to alter the excitability of the PN. As seen in Figure 3, TBOA dramatically influenced PN firing behaviour, particularly in the $100 \mathrm{~ms}$ period immediately after the burst stimulation when TBOA-enhanced depolarisation was at its maximum (see Figure 2). Although this short lasting burst firing behaviour was reminiscent of $\mathrm{CF}$ activation the responses remained graded and did not exhibit the all-or-none behaviour characteristic of CF activation. The enhanced PN firing also persisted during the time when the mGluR1 and EAAT4 dependent current were active (Figure 3). It was therefore surprising that blockade of mGluR1 did little to influence PN firing (Figure 3C). This suggests that the small remaining slow CNQX sensitive current (presumed to be driven by EAAT4) is sufficient to drive the membrane potential above the PN action potential threshold. It is possible, since EAAT4 is uniquely expressed in the PN dendrites $[12,13]$ that this glutamatergic current exerts a far more powerful contribution in the dendrite and only appears small and slow from our recordings at the soma. Alternatively, or in addition, since TBOA blocks the EAAT4 chloride conductance this may directly enhance PN dendrite excitability to sustain bursting behaviour.

\section{Inhibition of glutamate uptake recruits PF presynaptic metabotropic glutamate receptors (mGluR4) to reduce glutamate release from PFs}

Blockade of glutamate transporters also enhanced the phenomenon of paired pulse facilitation, a form of short term plasticity displayed by the PF synapse [44]. At this synapse, enhanced PPF indicates reduced presynaptic calcium and reduced glutamate release. We propose that TBOA reduced glutamate release may indicate an inbuilt plasticity mechanism to conserve overall synaptic weight at the PFs under conditions of excess glutamate $[45,46]$. Indeed, depression of transporter current residual CF EPSCs in double EAAT4 EAAT1 knockout mice also suggests that excess glutamate reduces glutamate release at CF synapses [41]. Critically, the reduced glutamate release at PFs required inhibition of glutamate uptake AND high frequency bursts, indicative of an activity dependent mechanism. Of the possible candidates, neither NMDA nor mGluR1-mediated mechanisms influenced the TBOA-induced change in PPF, rather it was prevented by the mGluR4 antagonist MPPG, a finding consistent with the unique presence of mGluR4 at PFs $[37,38]$. These receptors reduce glutamate release from PFs by reducing calcium influx [47] and by directly interacting with the release machinery $[48,49]$. MPPG alone did not change the behaviour of the PF-PN synapse suggesting that the combination of excess glutamate (by TBOA) and highfrequency stimulation together enabled sufficient glutamate to reach the PF presynaptic mGluR4 receptors. Indeed, a previous study showed that several seconds of PF stimulation is needed to activate mGluR4 [50]. The important consequence of mGluR4 recruitment in the face of excess glutamate is to usefully weaken, or balance, the PF-PN synapse. It is tempting to speculate that a similar mechanism contributes to long term depression (LTD) at this synapse, a phenomenon traditionally linked to motor learning. Interestingly, an orally active mGluR4 positive allosteric modulator influences motor behaviour [51].

\section{Conclusions}

\section{Relevance for cerebellar ataxia}

Our findings predict that loss of glutamate transporter activity leads to excessive and mis-timed PN output and altered PF plasticity that could lead to defective cerebellar output. Such changes are likely to take place during pre-symptomatic or early stages of a variety of human ataxias and mouse models of human ataxias [2-7]. Our results imply that chronic loss of transporter activity during progression of ataxia will be accompanied by hyperexcitability, oxidative stress, excitotoxicity and death of PNs. Since PN death is the end point of the disease novel therapeutic approaches to sustain EAAT transporter activity in the early phases of the disease merit serious attention. Possible approaches include reduced expression of GTRAP3-18, a protein that normally reduces EAAT3 activity [23], activation of innate compensatory mechanisms to elevate glutamate uptake [52] or selective stimulation of JAK kinases known to elevate EAATs [53], all of which may hold promise as future treatment strategies.

\section{Methods}

Mice

We used male C57 B6 mice aged 22-30 days. All animal husbandry and ethical procedures minimized animal suffering and followed internationally recognized standards as part of University of Otago approved guidelines in accordance with New Zealand Animal Welfare Act (1999).

\section{Electrophysiology}

$300 \mu \mathrm{m}$ thick saggital slices from the cerebellar vermis were prepared from mice following rapid anaesthesia with carbon dioxide. Slices were maintained at $24^{\circ} \mathrm{C}$ (TC324B, Harvard Apparatus, USA) in a flow ( $3 \mathrm{ml} /$ min) of artificial cerebrospinal fluid (aCSF), equilibrated 
with $95 \% \mathrm{O}_{2}$ and $5 \% \mathrm{CO}_{2}$, containing (in $\mathrm{mM}$ ) $\mathrm{NaCl}$ 126, $\mathrm{KCl}$ 2.5, $\mathrm{NaH}_{2} \mathrm{PO}_{4}$ 1.2, $\mathrm{MgCl}_{2}$ 1.3, $\mathrm{CaCl}_{2}$ 2.4, $\mathrm{NaHCO}_{3} 26$, glucose 10 . Whole cell recordings from PN soma under visual control used glass electrodes (3-5 $\mathrm{M} \Omega$ ) containing (in $\mathrm{mM}$ ) $\mathrm{KCl} 4.5, \mathrm{KOH} \mathrm{20,} \mathrm{MgCl}_{2} 3.48$, $\mathrm{NaCl} 4$, K gluconate 120 , Hepes 10 , sucrose $8, \mathrm{Na}_{2} \mathrm{ATP}$ 4, $\mathrm{Na}_{3}$ GTP 0.4, $10 \mathrm{mM}$ EGTA pH 7.3 and osmolarity $305 \mathrm{mOsm} / \mathrm{l}$. Voltage clamp (Axopatch 200B, Molecular Devices, USA) maintained cells at $-70 \mathrm{mV}$ holding current for at least 5 minutes prior to electrophysiological protocols (PClamp, Molecular Devices, USA). Mean uncompensated series resistance was $18 \pm 1.1 \mathrm{M} \Omega$ in control and $17.5 \pm 1.2 \mathrm{M} \Omega$ in $50 \mu \mathrm{M}$ TBOA, $\mathrm{P}>0.05$, $\mathrm{n}=12$, paired $t$-test; TBOA also did not influence $\mathrm{PN}$ input resistance or holding current, mean values were unchanged from $85.2 \pm 3$ to $87.6 \pm 3.7 \mathrm{M} \Omega$ and from $-154.7 \pm 21.9$ pA to $-188.8 \pm 29.7 \mathrm{pA}$, both $\mathrm{P}>0.05, \mathrm{n}=$ 12 , paired $t$-test. PF stimulation $(10 \mu \mathrm{s}, 2-20 \mathrm{~V}$, constant voltage stimulator, Digitimer, UK). Capacitance and input resistance were estimated from the current change during a $5 \mathrm{mV}$ step. We used a pair of stimuli for PPF measurements separated by an inter-stimulus interval of $50 \mathrm{~ms}$, and also a burst stimulus delivered $550 \mathrm{~ms}$ post PPF stimulation, consisting of 10 stimuli at $200 \mathrm{~Hz}(5 \mathrm{~ms}$ interval) (see Figure 1). Both types of stimulus were delivered at $0.011 \mathrm{~Hz}$ (every 30 seconds) using a glass aCSFfilled monopolar stimulation electrode $(200-600 \mathrm{k} \Omega$ ) placed in the outer two thirds of the molecular layer approximately $120-150 \mu \mathrm{m}$ from the recorded cell and the burst stimulus followed the PPF stimulation Figure 1). Loose patch recordings (Axopatch 200B) from the soma of PNs used glass electrodes with a resistance of approximately $6 \mathrm{M} \Omega$ employing the same internal solution as above.

\section{Solutions and pharmacological treatments}

EPSCs were evoked in the presence of $50 \mu \mathrm{M}$ picrotoxin (Sigma-Aldrich, UK) and prevented by $20 \mu \mathrm{M}$ CNQX, (1,2,3,4-Tetrahydro-6-nitro-2,3-dioxo-benzo[f] quinoxaline-7-sulfonamide (Tocris Cookson). Electrophysiology protocols were repeated after 10-15 minutes bath application of TBOA (50 $\mu \mathrm{M}$, (Sigma-Aldrich) a broad spectrum inhibitor of glutamate transport (10-50 $\mu \mathrm{M}$, Tocris Cookson). Pharmacological tools included $0.2 \mathrm{mM}$ (S-)MCPG, (S)- $\alpha$-Methyl-4-carboxyphenylglycine and $25 \mu \mathrm{M}$ MPPG, (RS)- $\alpha$-Methyl-4-phosphonophenylglycine all stored as a $1000 \times$ stock solution in DMSO or water. (See Table 1 for list of antagonists used).

Control applications of vehicle instead of TBOA over the same time period revealed no significant changes in any of our measured parameters $(\mathrm{P}>0.05, \mathrm{n}=4$, paired $\mathrm{t}$-tests).

\section{Analysis and statistics}

Offline analysis of all electrophysiological data used PClamp 9 (Molecular Devices, USA). Slow inward current amplitudes were measured at $500 \mathrm{~ms}$ after the onset of the high frequency stimulation, time constants of recovery of EPSCs used a single exponential fit. Statistical analysis was performed in Prism 6.0 (GraphPad) and Student's $t$-test and one and two way ANOVAs as appropriate.

\section{Abbreviations}

aCSF: Artificial cerebrospinal fluid; AMPA-R: Alpha-Amino-3-hydroxy-5-methyl4-isoxazolepropionic acid receptors; CF: Climbing fibres; CNQX: 6-cyano-7nitroquinoxaline-2,3-dione; EAAT: Excitatory amino acid transporter; GTRAP: Glutamate transporter associated protein; KA-R: Kainate receptors; MCPG: (S)-a-Methyl-4-carboxyphenylglycine; mGluR: Metabotropic glutamate receptor; MPPG: (RS)-a-Methyl-4-phosphonophenylglycine; PF: Parallel fibre; PN: Purkinje neuron; PPF: Paired pulse facilitation; SCA: Spinocerebellar ataxia; TBOA: DL-threo-beta-Benzyloxyaspartic acid.

\section{Competing interests}

The authors declare that they have no competing interests.

\section{Authors' contributions}

EMP and RME conceived the work; EMP completed all the experimental work; EMP and RME analysed the data; EMP and RME wrote the manuscript and have both read and approved the final manuscript.

\section{Acknowledgements}

EMP receives a University of Otago PhD Scholarship, work also supported by a University of Otago Dean's Bequest Grant.

Received: 17 December 2013 Accepted: 19 March 2014

Published: 16 June 2014

\section{References}

1. Rothstein JD, Martin L, Levey Al, Dykes-Hoberg M, Jin L, Wu D, Nash N, Kund RW: Localization of neuronal and glial glutamate transporters. Neuron 1994, 13:713-725.

2. Shigeri $Y$, Shimamoto $K$, Yasuda-Kamatani $Y$, Seal RP, Yumoto N, Nakajima T, Amara SG: Effects of threo-beta-hydroxyaspartate derivatives on excitatory amino acid transporters (EAAT4 and EAAT5). J Neurochem 2001, 79:297-302.

3. Fairman WA, Vandenberg RJ, Arriza JL, Kavanaugh MP, Amara SG: An excitatory amino-acid transporter with properties of a ligand-gated chloride channel. Nature 1995, 375:599-603.

4. Winter N, Kovermann P, Fahlke C: A point mutation associated with episodic ataxia 6 increases glutamate transporter anion currents. Brain 2012, 135:3416-3425.

5. de Vries B, Mamsa H, Stam AH, Wan J, Bakker SL, Vanmolkot KR, Haan J, Terwindt GM, Boon EM, Howard BD, Frants RR, Baloh RW, Ferrari MD, Jen JC, van den Maagdenberg AM: Episodic ataxia associated with EAAT1 mutation C186S affecting glutamate reuptake. Arch Neurol 2009, 66:97-101.

6. Jen JC, Wan J, Palos TP, Howard BD, Baloh RW: Mutation in the glutamate transporter EAAT1 causes episodic ataxia, hemiplegia, and seizures. Neurology 2005, 65:529-534.

7. Serra HG, Byam CE, Lande JD, Tousey SK, Zoghbi HY, Orr HT: Gene profiling links SCA1 pathophysiology to glutamate signaling in Purkinje cells of transgenic mice. Hum Mol Genet 2004, 13:2535-2543.

8. Lin X, Antalffy B, Kang D, Orr HT, Zoghbi HY: Polyglutamine expansion down-regulates specific neuronal genes before pathologic changes in SCA1. Nat Neurosci 2000, 3:157-163.

9. Custer SK, Garden GA, Gill N, Rueb U, Libby RT, Schultz C, Guyenet SJ, Deller T, Westrum LE, Sopher BL, La Spada AR: Bergmann glia expression of polyglutamine-expanded ataxin-7 produces neurodegeneration by impairing glutamate transport. Nat Neurosci 2006, 9:1302-1311.

10. Perkins EM, Clarkson YL, Sabatier N, Longhurst DM, Millward CP, Jack J, Toraiwa J, Watanabe M, Rothstein JD, Lyndon AR, Wyllie DJ, Dutia MB, Jackson M: Loss of beta-III spectrin leads to Purkinje cell dysfunction recapitulating the behavior and neuropathology of spinocerebellar ataxia type 5 in humans. J Neurosci 2010, 30:4857-4867.

11. Watase $K$, Hashimoto $K$, Kano M, Yamada K, Watanabe M, Inoue Y, Okuyama S, Sakagawa T, Ogawa S, Kawashima N, Hori S, Takimoto M, Wada K, Tanaka K: Motor discoordination and increased susceptibility to cerebellar injury in GLAST mutant mice. Eur J Neurosci 1998, 10:976-988. 
12. Furuta A, Martin $\sqcup$, Lin CL, Dykes-Hoberg M, Rothstein JD: Cellular and synaptic localization of the neuronal glutamate transporters excitatory amino acid transporter 3 and 4. Neuroscience 1997, 81:1031-1042.

13. Dehnes Y, Chaudhry FA, Ullensvang K, Lehre KP, Storm-Mathisen J, Danbolt NC: The glutamate transporter EAAT4 in rat cerebellar Purkinje cells: a glutamate-gated chloride channel concentrated near the synapse in parts of the dendritic membrane facing astroglia. J Neurosci 1998, 18:3606-3619.

14. Takayasu Y, lino M, Takatsuru Y, Tanaka K, Ozawa S: Functions of glutamate transporters in cerebellar Purkinje cell synapses. Acta Physiol (Oxf) 2009, 197:1-12

15. Torres-Salazar D, Fahlke C: Neuronal glutamate transporters vary in substrate transport rate but not in unitary anion channel conductance. J Biol Chem 2007, 282:34719-34726.

16. Paukert M, Huang YH, Tanaka K, Rothstein JD, Bergles DE: Zones of enhanced glutamate release from climbing fibers in the mammalian cerebellum. J Neurosci 2010, 30:7290-7299.

17. Sachs AJ, David SA, Haider NB, Nystuen AM: Patterned neuroprotection in the Inpp4a(wbl) mutant mouse cerebellum correlates with the expression of Eaat4. PLoS One 2009, 4:e8270.

18. Tsai MC, Tanaka K, Overstreet-Wadiche L, Wadiche Jl: Neuronal glutamate transporters regulate glial excitatory transmission. J Neurosci 2012, 32:1528-1535

19. Nikkuni O, Takayasu Y, lino M, Tanaka K, Ozawa S: Facilitated activation of metabotropic glutamate receptors in cerebellar Purkinje cells in glutamate transporter EAAT4-deficient mice. Neurosci Res 2007, 59:296-303.

20. Brasnjo G, Otis TS: Neuronal glutamate transporters control activation of postsynaptic metabotropic glutamate receptors and influence cerebellar long-term depression. Neuron 2001, 31:607-616.

21. Wadiche Jl, Jahr CE: Patterned expression of Purkinje cell glutamate transporters controls synaptic plasticity. Nat Neurosci 2005, 8:1329-1334.

22. Satake S, Song SY, Konishi S, Imoto K: Glutamate transporter EAAT4 in Purkinje cells controls intersynaptic diffusion of climbing fiber transmitter mediating inhibition of GABA release from interneurons. Eur J Neurosci 2010, 32:1843-1853.

23. Aoyama K, Watabe M, Nakaki T: Modulation of neuronal glutathione synthesis by EAAC1 and its interacting protein GTRAP3-18. Amino Acids 2012, 42:163-169.

24. Watabe M, Aoyama K, Nakaki T: A dominant role of GTRAP3-18 in neuronal glutathione synthesis. J Neurosci 2008, 28:9404-9413.

25. Aoyama K, Wang F, Matsumura N, Kiyonari H, Shioi G, Tanaka K, Kinoshita C, Kikuchi-Utsumi K, Watabe M, Nakaki T: Increased neuronal glutathione and neuroprotection in GTRAP3-18-deficient mice. Neurobiol Dis 2012, 45:973-982.

26. Lehre KP, Danbolt NC: The number of glutamate transporter subtype molecules at glutamatergic synapses: chemical and stereological quantification in young adult rat brain. J Neurosci 1998, 18:8751-8757.

27. Takayasu $Y$, lino M, Ozawa S: Roles of glutamate transporters in shaping excitatory synaptic currents in cerebellar Purkinje cells. Eur J Neurosci 2004, 19:1285-1295.

28. Takayasu Y, lino M, Shimamoto K, Tanaka K, Ozawa S: Glial glutamate transporters maintain one-to-one relationship at the climbing fiber-Purkinje cell synapse by preventing glutamate spillover. J Neurosci 2006, 26:6563-6572

29. Marcaggi $P$, Billups $D$, Attwell D: The role of glial glutamate transporters in maintaining the independent operation of juvenile mouse cerebellar parallel fibre synapses. J Physiol 2003, 552:89-107.

30. Piochon C, Irinopoulou T, Brusciano D, Bailly Y, Mariani J, Levenes C: NMDA receptor contribution to the climbing fiber response in the adult mouse Purkinje cell. J Neurosci 2007, 27:10797-10809.

31. Huang $H$, Bordey $A$ : Glial glutamate transporters limit spillover activation of presynaptic NMDA receptors and influence synaptic inhibition of Purkinje neurons. J Neurosci 2004, 24:5659-5669.

32. Chadderton P, Margrie TW, Hausser M: Integration of quanta in cerebellar granule cells during sensory processing. Nature 2004, 428:856-860.

33. Brown SP, Brenowitz SD, Regehr WG: Brief presynaptic bursts evoke synapse-specific retrograde inhibition mediated by endogenous cannabinoids. Nat Neurosci 2003, 6:1048-1057.

34. Maejima T, Hashimoto K, Yoshida T, Aiba A, Kano M: Presynaptic inhibition caused by retrograde signal from metabotropic glutamate to cannabinoid receptors. Neuron 2001, 31:463-475.

35. Marcaggi $P$, Attwell D: Short- and long-term depression of rat cerebellar parallel fibre synaptic transmission mediated by synaptic crosstalk. J Physiol 2007, 578:545-550.
36. Casado M, Dieudonne S, Ascher P: Presynaptic N-methyl-D-aspartate receptors at the parallel fiber-Purkinje cell synapse. Proc Natl Acad Sci U S A 2000, 97:11593-11597.

37. Mateos JM, Azkue J, Sarria R, Kuhn R, Grandes P, Knopfel T: Localization of the mGlu4a metabotropic glutamate receptor in rat cerebellar cortex. Histochem Cell Biol 1998, 109:135-139.

38. Abitbol K, Acher F, Daniel H: Depression of excitatory transmission at PF-PC synapse by group III metabotropic glutamate receptors is provided exclusively by mGluR4 in the rodent cerebellar cortex. J Neurochem 2008, 105:2069-2079.

39. Neale SA, Garthwaite J, Batchelor AM: Metabotropic glutamate receptor subtypes modulating neurotransmission at parallel fibre-Purkinje cell synapses in rat cerebellum. Neuropharmacology 2001, 41:42-49.

40. Delaney AJ, Jahr CE: Kainate receptors differentially regulate release at two parallel fiber synapses. Neuron 2002, 36:475-482.

41. Huang YH, Dykes-Hoberg M, Tanaka K, Rothstein JD, Bergles DE: Climbing fiber activation of EAAT4 transporters and kainate receptors in cerebellar Purkinje cells. J Neurosci 2004, 24:103-111.

42. Mim C, Balani P, Rauen T, Grewer C: The glutamate transporter subtypes EAAT4 and EAATs 1-3 transport glutamate with dramatically different kinetics and voltage dependence but share a common uptake mechanism. J Gen Physiol 2005, 126:571-589.

43. Melzer N, Torres-Salazar D, Fahlke C: A dynamic switch between inhibitory and excitatory currents in a neuronal glutamate transporter. Proc Natl Acad Sci U S A 2005, 102:19214-19218.

44. Mintz IM, Sabatini BL, Regehr WG: Calcium control of transmitter release at a cerebellar synapse. Neuron 1995, 15:675-688.

45. Royer S, Pare D: Conservation of total synaptic weight through balanced synaptic depression and potentiation. Nature 2003, 422:518-522.

46. Loebel A, Le Be JV, Richardson MJ, Markram H, Herz AV: Matched pre- and post-synaptic changes underlie synaptic plasticity over long time scales. J Neurosci 2013, 33:6257-6266.

47. Abitbol K, McLean H, Bessiron T, Daniel H: A new signalling pathway for parallel fibre presynaptic type 4 metabotropic glutamate receptors (mGluR4) in the rat cerebellar cortex. J Physio/ 2012, 590:2977-2994.

48. Ramos C, Chardonnet S, Marchand CH, Decottignies P, Ango F, Daniel H, Le Marechal P: Native presynaptic metabotropic glutamate receptor 4 (mGluR4) interacts with exocytosis proteins in rat cerebellum. $J$ Biol Chem 2012, 287:20176-20186.

49. Nakajima Y, Mochida S, Okawa K, Nakanishi S: Ca2 +-dependent release of Munc18-1 from presynaptic mGluRs in short-term facilitation. Proc Natl Acad Sci U S A 2009, 106:18385-18389.

50. Lorez M, Humbel U, Pflimlin MC, Kew JN: Group III metabotropic glutamate receptors as autoreceptors in the cerebellar cortex. $\mathrm{Br} J$ Pharmacol 2003, 138:614-625

51. East SP, Bamford S, Dietz MG, Eickmeier C, Flegg A, Ferger B, Gemkow MJ, Heilker R, Hengerer B, Kotey A, Loke P, Schanzle G, Schubert HD, Scott J, Whittaker M, Williams M, Zawadzki P, Gerlach K: An orally bioavailable positive allosteric modulator of the mGlu4 receptor with efficacy in an animal model of motor dysfunction. Bioorg Med Chem Lett 2010, 20:4901-4905.

52. Takatsuru Y, Eto K, Kaneko R, Masuda H, Shimokawa N, Koibuchi N, Nabekura J: Critical role of the astrocyte for functional remodeling in contralateral hemisphere of somatosensory cortex after stroke. J Neurosci 2013, 33:4683-4692.

53. Hosseinzadeh Z, Bhavsar SK, Sopjani M, Alesutan I, Saxena A, DermakuSopjani M, Lang F: Regulation of the glutamate transporters by JAK2. Cell Physiol Biochem 2011, 28:693-702.

doi:10.1186/2053-8871-1-3

Cite this article as: Power and Empson: Functional contributions of glutamate transporters at the parallel fibre to Purkinje neuron synapserelevance for the progression of cerebellar ataxia. Cerebellum \& Ataxias 2014 1:3 